\title{
Determinação de cádmio, cobre, cromo, níquel, chumbo e zinco em fosfatos de rocha
}

\author{
Mari Lucia Campos ${ }^{(1)}$, Francisco Nildo da Silva ${ }^{(1)}$, Antônio Eduardo Furtini Neto(1), \\ Luiz Roberto Guimarães Guilherme ${ }^{(1)}$, João José Marques ${ }^{(1)}$ e Alessandra Silveira Antunes ${ }^{(1)}$
}

\begin{abstract}
(1)Universidade Federal de Lavras, Dep. de Ciência do Solo, Caixa Postal 37, CEP 37200-000 Lavras, MG. E-mail: marilucia72@yahoo.com.br, fnildos@hotmail.com, furtini@ufla.br, guilherm@ufla.br, jmarques@ufla.br
\end{abstract}

\begin{abstract}
Resumo - A preocupação com metais pesados provenientes de fertilizantes minerais deve-se à possibilidade de entrada desses elementos na cadeia alimentar. O objetivo deste trabalho foi determinar o teor de metais pesados e comparar três métodos de extração (Embrapa, USEPA 3051A e USEPA 3050B) de Cd, Cr, Cu, Ni, Pb e Zn em fosfatos de rochas nacionais e importados. A quantificação dos teores desses elementos foi feita em espectrofotômetro de absorção atômica de chama ar-acetileno. Entre os fosfatos estudados, o termofosfato apresentou concentrações significativamente superiores de $\mathrm{Cr}$, $\mathrm{Cu}$, $\mathrm{Ni}$ e $\mathrm{Zn}$ e o fosfato natural 2, de $\mathrm{Cu}, \mathrm{Ni}, \mathrm{Pb}$ e $\mathrm{Zn}$. O fosfato reativo 3 apresentou a maior quantidade de $\mathrm{Cd}\left(145 \pm 13 \mathrm{mg} \mathrm{kg}^{-1}\right)$ e o fosfato natural 2, a maior quantidade de $\mathrm{Pb}\left(234 \pm 9 \mathrm{mg} \mathrm{kg}^{-1}\right)$. Os métodos avaliados podem ser aplicados em estudos de teor de metais pesados em fertilizantes fosfatados.
\end{abstract}

Termos para indexação: concentração, extração, metais pesados, fertilizantes.

\section{Determination of cadmium, copper, chromium, nickel, lead and zinc in rock phosphates}

\begin{abstract}
The possibility of food chain transfer is a matter of concern on studies related to heavy metals in mineral fertilizers. The objective of this paper was to determine the content of heavy methods and to compare three extraction procedures (Embrapa, 1999; USEPA 3051A and USEPA 3050B) for Cd, Cr, Cu, Ni, Pb, and Zn in national and imported rock phosphates. The quantification of the trace elements content was performed by airacetylene flame atomic absorption spectroscopy. Among the studied phosphates, the thermophosphate presented significantly greater concentrations of $\mathrm{Cd}, \mathrm{Cr}, \mathrm{Cu}, \mathrm{Ni}$, and $\mathrm{Zn}$ whereas $\mathrm{Cu}, \mathrm{Ni}, \mathrm{Pb}$, and $\mathrm{Zn}$ were found in greater contents in the natural phosphate 2 . The reactive phosphate 3 presented the greatest quantity of $\mathrm{Cd}$ $\left(145 \pm 13 \mathrm{mg} \mathrm{kg}^{-1}\right)$ and the natural phosphate 2, the highest quantity of $\mathrm{Pb}\left(234 \pm 9 \mathrm{mg} \mathrm{kg}^{-1}\right)$. The tested methods can be applied in studies concerning heavy metals in phosphate fertilizers.
\end{abstract}

Index terms: concentration, extraction, heavy metal, fertilizers.

\section{Introdução}

Insumos agrícolas ou subprodutos usados com finalidade corretiva ou nutricional na agricultura podem ser também uma fonte de contaminação com metais pesados. Adições globais de metais pesados ao solo por fertilizantes são da ordem de $30.000-250.000 \mathrm{~kg} \mathrm{ano}^{-1}$ de $\mathrm{Cd}, 50.000-580.000 \mathrm{~kg}^{2} \mathrm{ano}^{-1}$ de $\mathrm{Cu}, 30.000$ $380.000 \mathrm{~kg}$ ano ${ }^{-1} \mathrm{de} \mathrm{Cr}, 200.000-550.000 \mathrm{~kg}^{-1} \mathrm{ano}^{-1} \mathrm{de} \mathrm{Ni}$, 420.000-2.300.000 kg ano-1 de Pb e 260.000$1.100 .000 \mathrm{~kg} \mathrm{ano}^{-1}$ de Zn (Nriagu \& Pacyna, 1988).

Fertilizantes fosfatados contêm metais pesados da rocha que os originou ou dos ingredientes usados na industrialização do fertilizante. Metais pesados podem persistir no solo, por causa da sua baixa mobilidade, ou serem absorvidos pelas plantas e ingressarem na cadeia alimentar (Camelo et al., 1990; Abdel-Haleem et al., 2001).

Segundo Mortvedt (1987), as rochas fosfatadas usadas na produção dos fertilizantes são as maiores fontes de contaminação com Cd em solos agrícolas. Estima-se que a União Européia adiciona a seus solos cerca de 300 t ano $^{-1}$ de Cd (Huton \& Symon, 1986) e que a utilização de rochas fosfatadas da região oeste dos Estados Unidos resultou em adição de $100 \mathrm{~g} \mathrm{ha}^{-1} \mathrm{ano}^{-1}$ de Cd aos solos desse país (Mulla et al., 1980).

A alteração do teor natural de metais pesados nos solos pela aplicação de fertilizantes tende a ser obser- 
vada após décadas (McBride \& Spiers, 2001). Entretanto, é necessário avaliar o potencial de risco do uso de fertilizantes além de outras fontes de metais pesados, tais quais pulverizações com agroquímicos como sais de $\mathrm{Zn}$, arsenatos de $\mathrm{Cu}$ e $\mathrm{Pb}$, irrigação com água proveniente de rios que recebem grande carga poluidora, uso de lodo de esgoto, uso de composto de lixo urbano e esterco animal (Tiller, 1989).

O fosfato é um componente vital para o desenvolvimento vegetal, assim como para todos os seres vivos. Em plantas, o P é necessário na fotossíntese, respiração e reprodução (Stauffer \& Sulewski, 2003).

$\mathrm{O}$ aproveitamento do $\mathrm{P}$ pelas plantas é considerado baixo, já que apenas $10 \%$ da aplicação desse nutriente como fertilizante é aproveitado por culturas anuais. Além disso, as quantidades aplicadas superam as extrações pelas culturas, diferindo, neste aspecto, do $\mathrm{N}$ e do K, que apresentam relações mais estreitas entre aplicações nas adubações e extração pelas culturas, principalmente em produtividades elevadas. Essa diferença de comportamento tem sido atribuída à fixação de $\mathrm{P}$ pelos solos, que seria mais importante em solos tropicais, com elevados teores de óxidos de ferro e alumínio (Raij, 2003).

Somente no Brasil, o consumo de $\mathrm{P}_{2} \mathrm{O}_{5}$ em 2002 foi de 2.777.000 t (Lopes, 2003), sendo que, desse total, 43\% estavam na forma de monoamônio fosfato (MAP), $30 \%$ na forma de superfosfato simples (SS), $15 \%$ na forma de superfosfato triplo e $12 \%$ em outras fontes existentes no mercado.

A preocupação com metais pesados provenientes de fertilizantes minerais tradicionais ou de fonte alternativas, como lodos de tratamento biológico e demais resíduos industriais, relaciona-se sobretudo com a adubação de plantas empregadas diretamente na alimentação humana, como hortaliças e grãos (Alcarde \& Rodella, 2003). Essa preocupação leva em consideração elementos essenciais como $\mathrm{Fe}, \mathrm{Cu}, \mathrm{Zn}$ e $\mathrm{Mn}$, mas principalmente os elementos não essenciais às plantas como o $\mathrm{Pb}, \mathrm{Cr}$ e $\mathrm{Cd}$, que oferecem risco à saúde humana (ATSDR, 2002). Langenbach \& Sarpa (1985) avaliaram a concentração de $\mathrm{Cd}$ em onze fosfatos nacionais e observaram que eles continham menos que $2,0 \mathrm{mg} \mathrm{kg}^{-1} \mathrm{de}$ $\mathrm{Cd}$. Esses autores concluíram que essa baixa concentração nos fertilizantes fosfatados nacionais não oferecia risco para as plantas, animais e microorganismos do solo.

A rocha fosfatada Catalão apresenta $4 \mathrm{mg} \mathrm{kg}^{-1}$ de $\mathrm{Cd}, 19 \mathrm{mg} \mathrm{kg}^{-1}$ de $\mathrm{Cr}$ e $58 \mathrm{mg} \mathrm{kg}^{-1}$ de $\mathrm{Pb}$, enquanto o concentrado apatítico fino Araxá contém $7 \mathrm{mg} \mathrm{kg}^{-1}$ de
Cd, 44 mg kg $^{-1}$ de Cre 127 mg kg$^{-1}$ de Pb (Gabe \& Rodella, 1999, citados por Alcarde \& Rodella, 2003). Amaral Sobrinho et al. (1992) apresentam faixas típicas de metais pesados em fertilizantes fosfatados como sendo $0,1-170 \mathrm{mg} \mathrm{kg}^{-1}$ de $\mathrm{Cd}, 7-225 \mathrm{mg} \mathrm{kg}^{-1}$ de $\mathrm{Pb}$, 7-38 $\mathrm{mg} \mathrm{kg}^{-1}$ de $\mathrm{Ni}, 1-300 \mathrm{mg} \mathrm{kg}^{-1}$ de $\mathrm{Cu}$ e 50-1450 mg kg-1 de Zn. Até o momento, não há dados que permitam indicar níveis de tolerância desses elementos indesejáveis nos fertilizantes (Alcarde \& Rodella, 2003).

O conhecimento da concentração de elementos-traço em rochas fosfatadas e de métodos de extração eficientes é fundamental, especialmente em condições de agricultura intensiva e solos tropicais, onde há possibilidade de uso elevado de agroquímicos, como fertilizantes e corretivos.

O objetivo deste trabalho foi determinar o teor de Cd, $\mathrm{Cr}, \mathrm{Cu}, \mathrm{Ni}, \mathrm{Pb}$ e $\mathrm{Zn}$ em fosfatos de rochas brasileiras e estrangeiras e avaliar a eficiência de três métodos de extração de elementos-traço em fosfatos.

\section{Material e Métodos}

Foram avaliados os teores de Cd, Cr, Cu, Ni, Pb e Zn, pelos métodos de extração Embrapa (1999), USEPA 3050B (Usepa, 1998a) e USEPA 3051A (Usepa, 1998b), em oito fosfatos comercializados na região sul do Estado de Minas Gerais (Tabela 1). As amostras foram peneiradas em peneira de plástico de $1,5 \mathrm{~mm}$. A seguir foram trituradas até passar inteiramente por uma peneira descartável de náilon de 0,15 mm. Em ambas etapas, usou-se gral de ágata na trituração.

O método USEPA 3050B, da Agência de Proteção Ambiental dos EUA (Usepa, 1998a), utiliza aproximadamente $1 \mathrm{~g}$ de material que foi digerido por $10 \mathrm{~mL}$ de $\mathrm{HNO}_{3}$ concentrado em bloco digestor aberto por $10 \mathrm{mi}-$ nutos a $95 \pm 5^{\circ} \mathrm{C}$. As amostras foram resfriadas e após a adição de mais $5 \mathrm{~mL}$ de $\mathrm{HNO}_{3}$, novamente levadas ao

Tabela 1. Fosfatos de rocha avaliados.

\begin{tabular}{lllc}
\hline Fosfato & Sigla & Origem & $\begin{array}{c}\mathrm{P}_{2} \mathrm{O}_{5} \text { solúvel } \\
\text { ácido cítrico) }(\%)\end{array}$ \\
\hline Fosfato natural 1 & FN1 & Nacional & $<1^{(1)}$ \\
Fosfato natural 2 & FN2 & Nacional & $12^{(2)}$ \\
Fosfato reativo 1 & FR1 & Importado & $35^{(3)}$ \\
Fosfato reativo 2 & FR2 & Importado & $44^{(2)}$ \\
Fosfato reativo 3 & FR3 & Importado & $31^{(3)}$ \\
Fosfato reativo 4 & FR4 & Importado & - \\
Termofosfato & TF & Importado & $43^{(2)}$ \\
Superfosto triplo & ST & Nacional & $16^{(2)}$ \\
\hline
\end{tabular}

(1)Valor de solubilidade em água (Alcarde \& Rodella, 2003). (2)Vitti (2003). ${ }^{(3)}$ Sousa et al. (1999). 
bloco digestor para aquecimento a $95 \pm 5^{\circ} \mathrm{C}$ por mais 2 horas. Em seguida, foram resfriadas novamente e adicionou-se $2 \mathrm{~mL}$ de água destilada e $3 \mathrm{~mL}$ de $\mathrm{H}_{2} \mathrm{O}_{2}$ a $30 \%$. A seguir, foram novamente conduzidas ao bloco digestor por mais 2 horas a $95 \pm 5^{\circ} \mathrm{C}$. Por fim, as amostras foram resfriadas e receberam $5 \mathrm{~mL}$ de $\mathrm{HCl}$ e $10 \mathrm{~mL}$ de água destilada e foram aquecidas por 5 minutos a $95 \pm 5^{\circ} \mathrm{C}$. As alíquotas foram filtradas e armazenadas em recipiente de plástico.

O método USEPA 3051A (Usepa, 1998b) prevê a digestão de $0,5 \mathrm{~g}$ a 1,0 g de material em $10 \mathrm{~mL}$ de $\mathrm{HNO}_{3}$, concentrado em forno de microondas com tubos de Teflon PTFE à pressão de 0,76 MPa por 10 minutos. A temperatura alcançada à pressão de $0,76 \mathrm{MPa}$ é de aproximadamente $175^{\circ} \mathrm{C}$. Após a digestão, o extrato foi filtrado e seu volume completado para $40 \mathrm{~mL}$ com água destilada.

O método Embrapa (Embrapa, 1999) prevê a utilização de aproximadamente $0,5 \mathrm{~g}$ da amostra mais $20 \mathrm{~mL}$ de $\mathrm{HCl} 2 \mathrm{~mol} \mathrm{~L}^{-1}$, mantidas em fervura por 5 a 10 minutos em bloco digestor aberto. Após este período, as amostras foram resfriadas, filtradas, e as alíquotas foram transferidas para balão de $100 \mathrm{~mL}$; volume completado com água destilada.

Os métodos descritos não solubilizam totalmente a fração sólida dos fosfatos e são mais indicados que extrações totais para estudos de poluição do solo, pois representam o máximo potencialmente biodisponível de um dado poluente.

A quantificação dos teores de $\mathrm{Cd}, \mathrm{Cr}, \mathrm{Cu}, \mathrm{Fe}, \mathrm{Mn}, \mathrm{Ni}$, $\mathrm{Pb}$ e $\mathrm{Zn}$ nos extratos dos fertilizantes foi feita utilizando-se um espectrofotômetro de absorção atômica de chama ar-acetileno. As alíquotas obtidas nos três métodos de extração foram armazenadas em recipiente de plástico.

Todas as análises foram feitas em triplicata. Cada bateria de análise continha uma amostra de referência de teor do metal pesado conhecido e uma amostra em branco para fins de controle de qualidade. A análise de variância foi efetuada pelo software SISVAR e na comparação entre as médias foi utilizado o teste de ScottKnott a 5\% de probabilidade.

\section{Resultados e Discussão}

O termofosfato apresentou concentrações significativamente superiores de $\mathrm{Cr}$, Cu, Ni e $\mathrm{Zn}$ e o FN2, de Cu, $\mathrm{Ni}, \mathrm{Pb}, \mathrm{Zn}$ (Figura 1). Isso concorda em parte com os níveis obtidos por Amaral Sobrinho et al. (1992) em re- lação ao termofosfato Yoorin e o fosfato natural digerido em sistema aberto em $\mathrm{HClO}_{4}$ e $\mathrm{HF}$ na relação 1:5, apatita de Araxá, sendo que o primeiro era enriquecido em $\mathrm{Cr}$, $\mathrm{Ni}$ e $\mathrm{Pb}$, enquanto o segundo continha maiores teores de $\mathrm{Cd}$, Zn e Cu.

Os fosfatos nacionais (FN1 e FN2) apresentaram teor médio de $8,7 \mathrm{mg} \mathrm{kg}^{-1}$ de $\mathrm{Cd}$, ao passo que os fosfatos importados apresentaram, em média, $77 \mathrm{mg} \mathrm{kg}^{-1} \mathrm{de} \mathrm{Cd}$, ou seja, os fosfatos importados avaliados contêm aproximadamente nove vezes mais Cd que os fosfatos nacionais. O teor médio para FN2 de aproximadamente $215 \pm 19 \mathrm{mg} \mathrm{kg}^{-1}$ de $\mathrm{Pb}$ é seis vezes maior que o teor médio encontrado para os outros fosfatos avaliados neste estudo (35 $\pm 15 \mathrm{mg} \mathrm{kg}^{-1}$ ) (FR1, FR2, FR3, FR4, ST e TF), com exceção do FN1, que apresentou teor médio de $\mathrm{Pb}$ de $81 \pm 5 \mathrm{mg} \mathrm{kg}^{-1}$.

Os valores de $\mathrm{Cu}\left(4,2 \pm 0,2\right.$ a $\left.115 \pm 5 \mathrm{mg} \mathrm{kg}^{-1}\right), \mathrm{Pb}(17 \pm 2$ a $\left.234 \pm 9 \mathrm{mg} \mathrm{kg}^{-1}\right)$ e $\mathrm{Zn}\left(20 \pm 3\right.$ a $\left.1.013 \pm 44 \mathrm{mg} \mathrm{kg}^{-1}\right)$ (Figura 1) encontram-se dentro do intervalo apresentado por Kabata-Pendias \& Pendias (2001). Entretanto, os valores de $\mathrm{Cu}$ e $\mathrm{Pb}$ observados nos oito fosfatos estão acima do encontrado por Camelo et al. (1990), que incluiu em seu estudo dois fosfatos do Brasil.

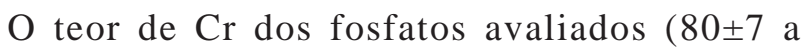
$514 \pm 42 \mathrm{mg} \mathrm{kg}^{-1}$ ) foi superior ao intervalo de valores apresentado por Camelo et al. (1990) para fertilizantes fosfatados (10-73 mg kg${ }^{-1}$ ) comercializados na Argentina. Entretanto, quando o intervalo de valores considerado é aquele apresentado por Kabata-Pendias \& Pendias (2001), 66-245 mg kg-1 de Cr, observa-se que sete dos oito fosfatos encontram-se dentro da faixa citada pelos autores, com exceção do termofosfato. Entre os fosfatos estudados por Camelo et al. (1990) estão dois superfosfatos do Brasil que apresentaram em média $25 \mathrm{mg} \mathrm{kg}^{-1} \mathrm{de} \mathrm{Cr}$, valor bem abaixo do observado para o superfosfato triplo avaliado ( $173 \pm 11 \mathrm{mg} \mathrm{kg}^{-1} \mathrm{de} \mathrm{Cr}$ ).

O teor de Ni dos fosfatos FN1, FR2, FR4 e ST encontra-se dentro do intervalo de valores apresentados por Kabata-Pendias \& Pendias (2001) para fertilizantes fosfatados, 7-38 $\mathrm{mg} \mathrm{kg}^{-1} \mathrm{de} \mathrm{Ni}$, enquanto os fosfatos FN2, FR1, FR3 e TF apresentaram teores de Ni maiores que $38 \mathrm{mg} \mathrm{kg}^{-1}$. O termofosfato apresentou o maior valor de Ni (258 $\left.\pm 22 \mathrm{mg} \mathrm{kg}^{-1}\right)$, o que concorda com o observado por Amaral Sobrinho et al. (1992). Porém, o teor de Ni encontrado por esses autores no termofosfato Yoorin foi 13 vezes maior ( $3.300 \mathrm{mg} \mathrm{kg}^{-1}$ ) que o observado no termofosfato avaliado. 
Os fosfatos de rocha apresentaram teores de Cd entre $5 \pm 0,6$ e $145 \pm 13 \mathrm{mg} \mathrm{kg}^{-1}$ (Figura 1). Esses valores
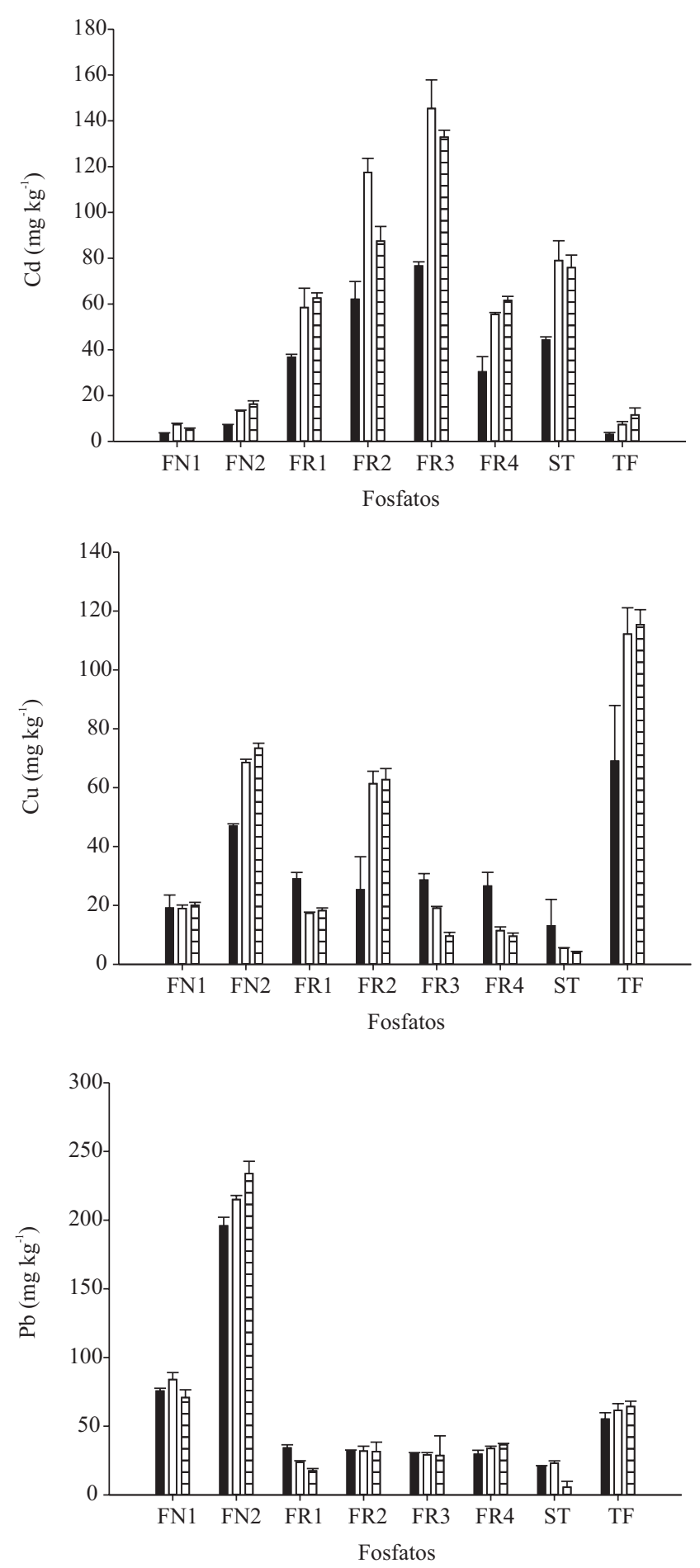

encontram-se dentro do intervalo citado por KabataPendias \& Pendias (2001) para fertilizantes fosfatados,
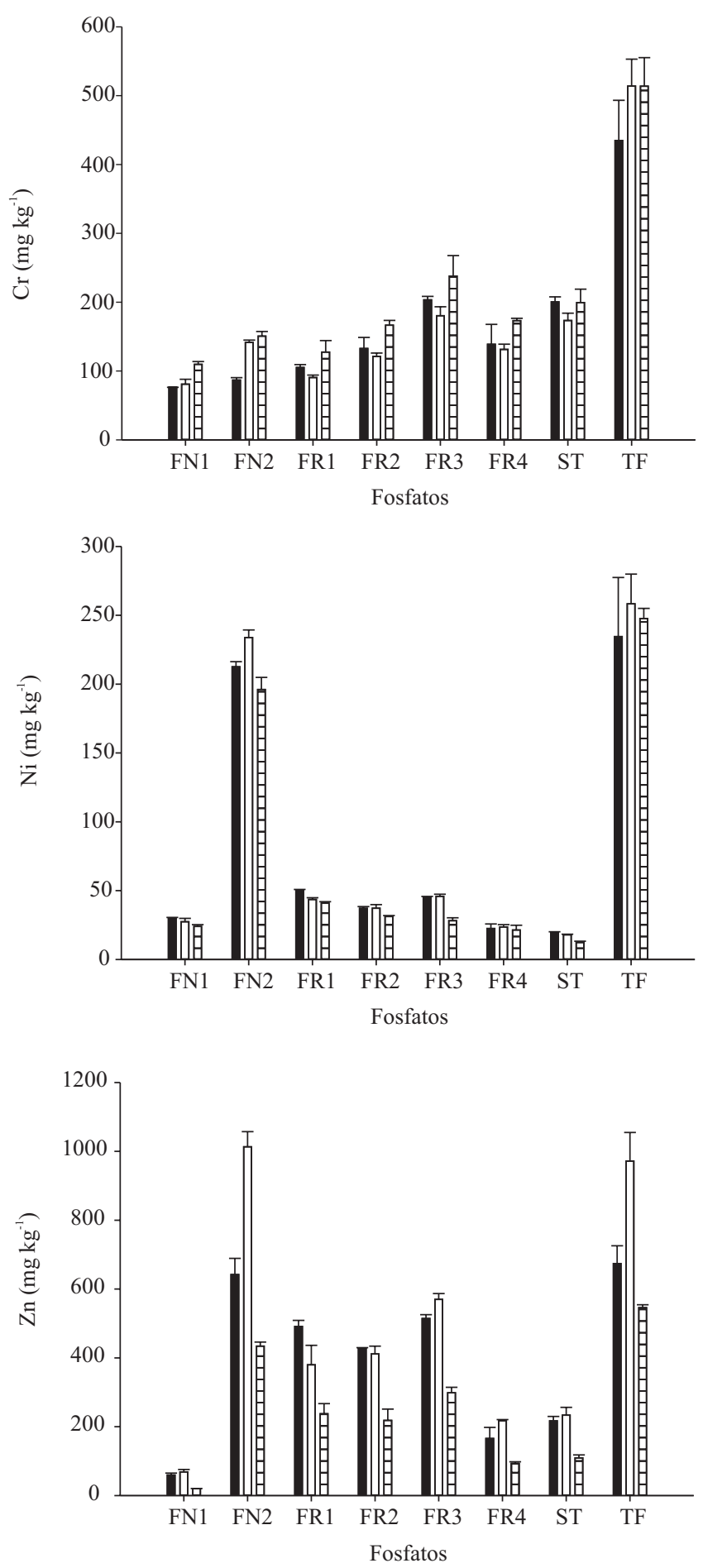

Figura 1. Teores médios de Cd, Cr, Cu, Ni, Pb e Zn em fosfatos nacionais naturais (FN1 e FN2), superfosfato triplo nacional (ST), fosfatos reativos importados (FR1, FR2, FR3 e FR4) e termofosfato importado (TF), para cada método de extração (USEPA 3051A, ఐ; USEPA 3050B, $\square$; Embrapa, 目). Os valores apresentados representam média de três repetições e as barras acima das colunas representam o desvio padrão em torno da média. 
0,1-170 $\mathrm{mg} \mathrm{kg}^{-1}$ de Cd. Entretanto, os teores de Cd estão acima daqueles encontrados por Amaral Sobrinho et al. (1992) para rochas apatíticas (2-7 $\mathrm{mg} \mathrm{kg}^{-1}$ de Cd). Segundo Holm et al. (1996) e Smolders et al. (1999), o uso de fertilizantes fosfatados, lodo de esgoto e a deposição atmosférica podem resultar em aumento gradual de Cd em solos agrícolas.

Calculou-se o incremento de Cd proporcionado pela aplicação de $200 \mathrm{~kg} \mathrm{ha}^{-1}$ de $\mathrm{P}_{2} \mathrm{O}_{5}$ do fosfato FR3 incorporado a $0,1 \mathrm{~m}$ de profundidade (Tabela 2 ). A quantidade de $200 \mathrm{~kg} \mathrm{ha}^{-1}$ de $\mathrm{P}_{2} \mathrm{O}_{5}$ baseia-se na quantidade de $\mathrm{P}_{2} \mathrm{O}_{5}$ exigida pela cultura do milho para produção de $7 \mathrm{t} \mathrm{ha}^{-1}$ (Ernani et al., 2000). Para o cálculo de incremento de elemento-traço no solo (Tabela 2), foram utilizados os valores de $\mathrm{P}_{2} \mathrm{O}_{5}$ solúveis apresentados na Tabela 1 . O incremento anual de Cd seria de 0,094 $\mathrm{mg} \mathrm{kg}^{-1}$, ou seja, seriam necessárias mais de 111 aplicações para atingir $10 \mathrm{mg} \mathrm{kg}^{-1} \mathrm{de} \mathrm{Cd}$, que é o valor de intervenção em solos agrícolas conforme Cetesb (2001). Por outro lado, em cerca de cinco anos, o conteúdo de Cd no solo provavelmente dobraria, já que o conteúdo natural de Cd na maioria dos solos é apenas $0,5 \mathrm{mg} \mathrm{kg}^{-1}$ (Campos et al., 2003).

A possibilidade de atingir a concentração máxima permitida, não só para Cd, mas também para os outros elementos avaliados, aumenta com o uso de quantidades maiores de fertilizantes fosfatados associados a lodo de esgoto e outros adubos que contenham elementostraço. Ramalho et al. (1999) avaliaram se a utilização de fertilizantes fosfatados e irrigação com água poluída promoveriam aumento nos teores de metais pesados no solo. Esses autores observaram que solos que receberam fertilizantes fosfatados durante 25 anos apresentaram aumento significativo de $\mathrm{Cd}\left(0,66 \mathrm{mg} \mathrm{kg}^{-1}\right)$ quando comparados com área controle $\left(0,5 \mathrm{mg} \mathrm{kg}^{-1}\right)$, sem, contudo, elevá-lo a níveis críticos. As condições que determinam a capacidade de adsorção, tais como pH, teor de

Tabela 2. Incremento de $\mathrm{Cd}, \mathrm{Cr}, \mathrm{Cu}, \mathrm{Ni}, \mathrm{Pb}$ e $\mathrm{Zn}$ proporcionado pela aplicação de $200 \mathrm{~kg}$ ha ${ }^{-1}$ de $\mathrm{P}_{2} \mathrm{O}_{5}$ incorporado em $0,1 \mathrm{~m}$ de profundidade.

\begin{tabular}{cccccr}
\hline Elemento & Fonte & $\begin{array}{c}\mathrm{P}_{2} \mathrm{O}_{5} \\
\text { solúvel } \\
(\%)\end{array}$ & $\begin{array}{c}\text { Concen- } \\
\text { tração } \\
\left(\mathrm{mg} \mathrm{kg}^{-1}\right)\end{array}$ & $\begin{array}{c}\text { Incremento } \\
\text { por aplicação } \\
\left(\mathrm{mg} \mathrm{kg}^{-1}\right)\end{array}$ & $\begin{array}{r}\text { Número de } \\
\text { aplicações }\end{array}$ \\
\hline $\mathrm{Cd}$ & $\mathrm{FR} 3$ & 31 & 10 & 0,094 & 415 \\
$\mathrm{Cr}$ & $\mathrm{TF}$ & 43 & 300 & 0,239 & 5.439 \\
$\mathrm{Cu}$ & $\mathrm{TF}$ & 43 & 100 & 0,054 & 28.302 \\
$\mathrm{Ni}$ & $\mathrm{TF}$ & 43 & 50 & 0,120 & 3.500 \\
$\mathrm{~Pb}$ & $\mathrm{FN} 2$ & 12 & 200 & 0,389 & 771 \\
$\mathrm{Zn}$ & $\mathrm{TF}$ & 43 & 500 & 0,452 & 6.194 \\
\hline
\end{tabular}

${ }^{(1)}$ Valores conforme Cetesb (2001). matéria orgânica, teor de argila e óxidos de $\mathrm{Fe}$ e $\mathrm{Al}$, afetam a disponibilidade e mobilidade dos metais pesados presentes nos fosfatos (Abdel-Haleem et al., 2001). É importante também considerar as condições locais que possam levar a perdas por erosão de partículas de solo enriquecidas em metais pesados para outras áreas ou para corpos d'água.

A Tabela 3 apresenta os teores médios de elementos-traço em oito fosfatos nacionais e importados, determinados pelos métodos USEPA 3051A, USEPA 3050B e Embrapa.

Quanto ao teor de $\mathrm{Cu}$, não houve diferença entre os métodos de extração (Tabela 3). Já para Ni e Zn, não houve diferença estatística entre os métodos USEPA 3051A e USEPA 3050B que foram superiores ao método Embrapa (1999).

Os métodos Embrapa e USEPA 3051A não diferiram estatisticamente entre si no tocante à extração de $\mathrm{Pb}$ e extraíram em torno de $30 \%$ mais $\mathrm{Pb}$ que o método USEPA 3050B. Campos et al. (2003) compararam a extração de $\mathrm{Pb}$ em 19 latossolos brasileiros com águarégia e USEPA 3051A e também observaram que o último chegou a extrair até $30 \%$ mais $\mathrm{Pb}$ que a águarégia. O método USEPA 3050B extraiu maior quantidade de Cd que os outros métodos, enquanto para $\mathrm{Cr}$, o método que mais extraiu foi o Embrapa.

A aplicação de procedimento de extração com ácidos fortes como água-régia, $\mathrm{HNO}_{3}$ ou $\mathrm{HCl}$ tem como objetivo determinar o potencial de disponibilidade e de mobilidade do poluente, o que está relacionado à sua transferência do sistema solo para a planta (Rauret, 1998). Os três métodos avaliados podem ser aplicados em estudos de teor dos elementos avaliados. Porém, métodos convencionais em bloco digestor aberto, como o USEPA 3050B, são laboriosos, além de resultar em possíveis perdas por volatilização, enquanto a extração em microondas não só é menos poluidora como tam-

Tabela 3. Teor médio de elementos-traço em fosfatos de rochas nacionais e importados determinados pelos métodos Embrapa, USEPA3051A e USEPA3050B ${ }^{(1)}$.

\begin{tabular}{lcccrcc}
\hline Método & $\mathrm{Cd}$ & $\mathrm{Cr}$ & $\mathrm{Cu}$ & $\begin{array}{c}\mathrm{Ni} \\
\mathrm{Pb}\end{array}$ & $\mathrm{Zn}$ \\
\hline Embrapa & $57 \mathrm{~b}$ & $210 \mathrm{a}$ & $39 \mathrm{a}$ & $75 \mathrm{~b}$ & $61 \mathrm{a}$ & $245 \mathrm{~b}$ \\
USEPA 3051A & $33 \mathrm{~b}$ & $172 \mathrm{~b}$ & $32 \mathrm{a}$ & $81 \mathrm{a}$ & $59 \mathrm{a}$ & $400 \mathrm{a}$ \\
USEPA 3050B & $93 \mathrm{a}$ & $157 \mathrm{~b}$ & $39 \mathrm{a}$ & $48 \mathrm{a}$ & $44 \mathrm{~b}$ & $348 \mathrm{a}$ \\
\hline
\end{tabular}

${ }^{(1)}$ Médias seguidas da mesma letra não diferem estatisticamente pelo teste de Scott-Knot a 5\% de probabilidade. 
bém resulta em menor perda de elementos por volatilização e secagem da solução extratora. Além disso, proporciona menor tempo de digestão, boa recuperação de muitos dos elementos voláteis, reduz o risco de contaminação externa e requer menor quantidade de ácidos (Bettinelli et al., 2000).

\section{Conclusões}

1. O termofosfato apresenta concentrações superiores de $\mathrm{Cr}$, $\mathrm{Cu}$, Ni e $\mathrm{Zn}$.

2. O fosfato reativo 3 apresenta a maior quantidade de Cd, enquanto o fosfato natural 2 apresenta a maior quantidade de $\mathrm{Pb}$.

3. Os três métodos avaliados podem ser aplicados em estudos de teor de metais pesados em fertilizantes fosfatados.

\section{Agradecimento}

Ao CNPq, pelas bolsas concedidas aos autores Antônio Eduardo Furtini Neto, Luiz Roberto Guimarães Guilherme e João José Marques.

\section{Referências}

ABDEL-HALEEM, A.S.; SCROON, A.; EL-BAHI, S.M.; ZOHNY, E. Heavy metals and rare earth elements in phosphate fertilizer components using instrumental neutron activation analysis. Applied Radiation and Isotopes, v.55, p.569-573, 2001.

ALCARDE, J.C.; RODELLA, A.A. Qualidade e legislação de fertilizantes e corretivos. In: CURI, N.; MARQUES, J.J.; GUILHERME, L.R.G.; LIMA, J.M.; LOPES, A.S.; ALVAREZ VENEGAS, V.H. Tópicos em Ciência do Solo. Viçosa: Sociedade Brasileira de Ciência do Solo, 2003. v.3, p.291-334.

AMARAL SOBRINHO, N.M.B.; COSTA, L.M., OLIVEIRA, C.; VELLOSO, A.C.X. Metais pesados em alguns fertilizantes e corretivos. Revista Brasileira de Ciência do Solo, v.16, p.271276, 1992.

ATSDR (Agency for Toxic Substances and Disease Control). CERCLA priority list of hazardous substances. Disponível em: <http://www.atsdr.cdc.gov/clist.html>. Acesso em: jun. 2002.

BETTINELLI, M.; BEONE, G.M.; SPEZIA, S.; BAFFI, C. Determination of heavy metals in soils and sediments by microwaveassisted digestion and inductively coupled plasma optical emission spectrometry analysis. Analytica Chimica Acta, v.424, p.289-296, 2000.

CAMELO, L.G.L.; MIGUEZ, S.R.; MARBÁN, L. Heavy metals input with phosphate fertilizers used in Argentina. The Science of the Total Environment, v.204, p.245-250, 1990.
CAMPOS, M.L.; PIERANGELI, M.A.P.; GUILHERME, L.R.G.; MARQUES, J.J.; CURI, N. Baseline concentration of heavy metals in Brazilian Latosols. Communications in Soil Science and Plant Analysis, v.34, p.547-558, 2003.

CETESB (Companhia de Tecnologia de Saneamento Ambiental). Relatório de estabelecimento de valores orientadores para solos e águas subterrâneas no Estado de São Paulo. São Paulo: Secretaria de Estado do Meio Ambiente, 2001. 230p.

EMBRAPA. Centro Nacional de Pesquisa de Solos (Rio de Janeiro, RJ). Manual de análise química dos solos, plantas e fertilizantes. Embrapa Solos, 1999. 370p.

ERNANI, R.P.; NASCIMENTO, J.A.L.; CAMPOS, M.L.; CAMILLO, R.J. Aumento da disponibilidade de fósforo no solo diminuiu a resposta do milho à calagem. Revista Brasileira de Ciência do Solo, v.24, p.537-544, 2000.

HOLM, P.E.; ANDERSEN, B.B.H.; CHRISTENSEN, T.H. Cadmium solubility in aerobic soils. Soil Science Society of America Journal, v.60, p.775-780, 1996.

HUTON, M.; SYMON, C. The quantities of cadmium, lead, mercury and arsenic entering the UK environment from human activities. Science of the Total Environment, v.57, p.129-150, 1986.

KABATA-PENDIAS, A.; PENDIAS, H. Trace elements in soils and plants. $3^{\text {rd }}$ ed. Boca Raton: CRC Press, 2001. 413p.

LANGENBACH, T.; SARPA, M. Teor de cádmio nos fertilizantes fosfatados brasileiros. Revista Brasileira de Ciência do Solo, v.9, p.179-181, 1985.

LOPES, A.S. Reservas de fosfatados e produção de fertilizantes fosfatados no Brasil e no mundo. In: SIMPÓSIO SOBRE FÓSFORO NA AGRICUltURA BRASILEIRA, 2003, Piracicaba. Anais. Piracicaba: Potafos/Anda, 2003. 1 CD-ROM.

MCBRIDE, M.B.; SPIERS, G. Trace elements content of selected fertilizers and dairy manures as determined by ICP-MS. Communications in Soil Science and Plant Analysis, v.32, p.139156, 2001.

MORTVEDT, J.J. Cadmium levels in soils and plants from some long-term soil fertility experiments in United States of America. Journal of Environmental Quality, v.16, p.137-142, 1987.

MULLA, D.J.; PAGE, A.L.; GANGE, T.J. Cadmium accumulations and bioavailability in soils from long-term phosphorus fertilization. Journal of Environmental Quality, v.9, p.408-412, 1980.

NRIAGU, J.O.; PACYNA, J.M. Quantitative assessment of worldwide contamination of air, water and soils with trace metals. Nature, v.333, p.134-139, 1988.

RAIJ, B. van. Fósforo no solo e interação com outros elementos. In: SIMPÓSIO SOBRE FÓSFORO NA AGRICULTURA BRASILEIRA, 2003, Piracicaba. Anais. Piracicaba: Potafos/Anda, 2003. 1 CD-ROM.

RAMALHO, J.F.G.P.; AMARAL SOBRINHO, N.M.B.; VELLOSO, A.C.X. Acúmulo de metais pesados em solos cultivados com cana-de-açúcar pelo uso contínuo de adubação fosfatada e água de irrigação. Revista Brasileira de Ciência do Solo, v.23, p.971979, 1999. 
RAURET, G. Extraction procedures for the determination of heavy metals in contaminated soil and sediment. Talanta, v.46, p.449-455, 1998.

SMOLDERS, E.; BRANS, K.; FOLDI, A.; MERCK, R. Cadmium fixation in soils measured by isotopic dilution. Soil Science Society of American Journal, v.63, p.78-86, 1999.

SOUSA, D.M.G. de; REIN, T.A.; LOBATO, E.; SOARES, W. Eficiência agronômica de fosfatos naturais reativos na região dos cerrados. In: CONGRESSO BRASILEIRO DE CIÊNCIA DO SOLO, 27., 1999, Brasília, DF. Ciência do solo e qualidade de vida. Planaltina: Embrapa Cerrados, 1999. 1 CD-ROM.

STAUFFER, M.D.; SULEWSKI, G. Phosphorus: essential for life. In: SIMPÓSIO SOBRE FÓSFORO NA AGRICULTURA
BRASILEIRA, 2003, Piracicaba. Anais. Piracicaba: Potafos/Anda, 2003. 1 CD-ROM.

TILLER, K.G. Heavy metals in soils and their environmental significance. In:__ Advances in Soil Science. New York: SpringerVerlag, 1989. v.9, p.113-114.

USEPA. Method 3050 B. 1998a. Disponível em: http:// www.epa.gov/SW-846/pdfs/3050b.pdf. Acesso em: fev. 2004.

USEPA. Method 3051 A. 1998b. Disponível em: http:// www.epa.gov/SW-846/3051a.pdf. Acesso em: mar. 2004.

VITTI, G.C. Eficiência agronômica de termofosfatos e fosfatos alternativos. In: SIMPÓSIO SOBRE FÓSFORO NA AGricultura Brasileira, 2003, Piracicaba. Anais. Piracicaba: Potafos/Anda, 2003. 1 CD-ROM.

Recebido em 23 de abril de 2004 e aprovado em 18 de novembro de 2004 